\title{
Combination of Geometric and Orthometric Heights in the Presence of Geoid and Quasi-geoid Models
}

\author{
C. Kotsakis and I. Tsalis
}

\begin{abstract}
A generalization of the well-known relationship between geometric and orthometric heights is presented in this paper. The advantage of our revised formulation is that, instead of the non-determinable true orthometric heights, it employs the (most commonly used in geodetic practice) Helmert orthometric heights. Based on standard concepts from physical geodesy theory and straightforward analytical derivations, we obtain a set of linearized constraints relating the geometric and Helmert orthometric heights in the presence of a geoid or quasigeoid model. These constraints should be theoretically satisfied in the case of errorless data and thus they provide a standard framework for the joint analysis and the quality testing of heterogeneous heights in a terrestrial network.
\end{abstract}

\section{Keywords}

Geoid • Quasi-geoid • Helmert orthometric heights • Vertical reference frame $\bullet$ Height transformation

\section{Introduction}

Since the advent of space-geodetic positioning techniques, the joint analysis of heterogeneous height data has become an important tool for many geodetic applications. The most prominent example is the combination of GPS derived geometric heights with spirit levelled orthometric heights in the presence of a geoid model, which has been a standard method for the quality testing of global geopotential models (or terrestrial leveling networks) and the implementation of unification schemes between traditional and geoid-based vertical datums (e.g. Rapp 1994; Pan and Sjöberg 1998; Burša et al. 2004; Sánchez 2007; Amos and Featherstone 2009; Kotsakis and Katsambalos 2010). The

C. Kotsakis $(\bowtie) \bullet \mathrm{I}$. Tsalis

Department of Geodesy and Surveying, Aristotle University

of Thessaloniki, Thessaloniki, 541 24, Greece

e-mail: kotsaki@topo.auth.gr underlying principle relies on the low-order parameterization of the theoretical constraint (Heiskanen and Moritz 1967, p. 187)

$$
h-N-H=0
$$

and the assessment of the results from its least squares adjustment over a network of GPS/leveling benchmarks. For most applications the emphasis is given on the analysis of the adjusted residuals in terms of (i) their statistical characteristics that indicate the combined accuracy level of the different height types, and (ii) their spatial modeling that facilitates the identification of local systematic distortions in the geoid model and/or the leveled orthometric heights. The estimated parameters from the aforementioned adjustment do not usually carry any theoretical or practical relevance, other than providing the overall trend of the heterogeneous height differences in the underlying network due to hidden biases and long-wavelength data errors. Indeed, the trend modeling schemes that are used in practice for the analysis of the height differences $h-N-H$ are mostly empirical (e.g. bias/tilt models, polynomial surfaces, Fotopoulos 2003) without considering 
any geodetically important principles for vertical reference systems and their realizations from different data sources. The aim of this paper is to revise the theoretical constraint between geometric, orthometric and geoid heights beyond the simplified form of Eq. (1), thus providing a general parametric model that can be used for the joint analysis of heterogeneous height data in terrestrial networks. Our treatment is confined to a theoretical discussion of the basic principles and the motivation for such a revised formulation in the context of the combination problem of Helmert-type orthometric heights not only with a geoid model (as it is usual case in the geodetic literature) but also with a quasi-geoid model.

\section{Fundamental Relationship Between Geometric and Orthometric Heights}

The theoretical equation relating the geometric and orthometric heights in the presence of a geoid model has the general form

$$
h-N-H=\frac{\delta W_{\mathrm{o}}}{g^{\left(W_{\mathrm{o}}\right)}}
$$

where the meaning of each height type is shown in Fig. 1. The term $\delta W_{\mathrm{o}}$ refers to the geopotential difference between (i) the local vertical datum (LVD) defining the zero-height level of the orthometric heights and (ii) the equipotential surface which is realized by the geoid model, whereas $g^{\left(W_{0}\right)}$ denotes the gravity value on the geoid as depicted in Fig. 1.

Essentially the above formula quantifies, within a linear approximation, the vertical separation between the equipotential surfaces $W=W_{\mathrm{o}}^{\mathrm{LVD}}$ and $W=W_{\mathrm{o}}$ using three different types of geodetic "observables". Its linearization error is negligible provided that the two surfaces are not separated by more than few meters, a fact that can be ensured for any reasonable geoid model in relation with most existing vertical datums. ${ }^{1}$ The non-parallelism of these equipotential surfaces is reflected through the presence of the non-constant gravity value $g^{\left(W_{0}\right)}$ in Eq. (2). In fact, the gravity variation on the geoid can cause an almost mm-level dispersion in the vertical offset $h-N-H$, mostly over very large distances $(>10,000 \mathrm{~km})$. For most practical applications, however, we may replace the geoidal gravity with a conventional mean gravity value ( $g$ or $\gamma$ ) without causing any notable modeling error in Eq. (2).

\footnotetext{
${ }^{1}$ If the geoid model does not include a zero-degree term then the $W_{\mathrm{o}}$ value is solely dictated by the normal gravity potential of the underlying reference ellipsoid and the mass difference between the actual Earth and the reference ellipsoid, i.e. $W_{\mathrm{o}}=f\left(U_{\mathrm{o}}, \delta M\right)$.
}

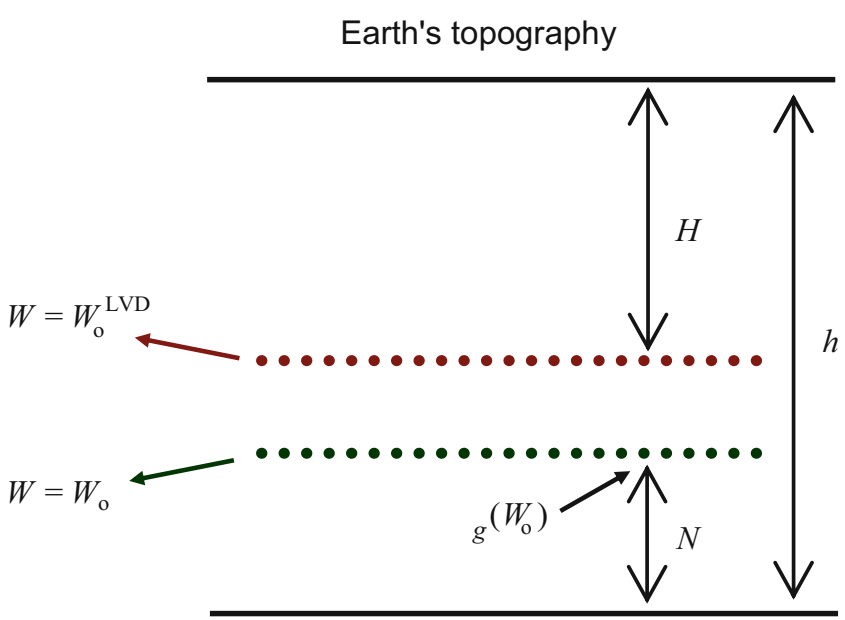

Ref. Ellipsoid

Fig. 1 The general relationship between the geometric and orthometric heights in the presence of a geoid model

The theoretical validity of the previous relationship relies on two basic assumptions. Firstly, the deflection of the vertical on the Earth's surface is ignored so that the geometric and orthometric heights can be treated as straight distances along the same normal direction. The approximation error in Eq. (2) due to this simplification remains below the mm level and it is thus negligible for most practical applications (Jekeli 2000, p. 15). Secondly, the geometric height and the geoid height should refer to a common geodetic reference system with respect to the same Earth reference ellipsoid so that Eq. (2) remains free of any datum related inconsistencies. This second assumption includes also the requirement that the permanent tide effect and other geophysical loading phenomena are treated and corrected in a consistent way among the heterogeneous height types.

\section{Revised Theoretical Constraint for Geometric and Orthometric Heights}

The (true) orthometric height is not a determinable physical quantity from geodetic measurements since it depends on the unknown mass distribution within the Earth. Therefore, it would be useful to replace Eq. (2) with a more realistic, yet equally rigorous, theoretical constraint which incorporates the operational type of Helmert orthometric heights.

Based on the definition of the true and Helmert orthometric heights (Heiskanen and Moritz 1967, Chap. 4), we have the following relationship between them

$$
H=\left(\frac{\bar{g}^{\text {helm }}}{\bar{g}}\right) H^{\text {helm }}
$$


where $\bar{g}$ denotes the average gravity along the actual plumbline between the Earth's surface and the zero-height reference surface of the vertical datum (i.e. $W=W_{\mathrm{o}}^{\mathrm{LVD}}$ ). The term $\bar{g}^{\text {helm }}$ corresponds to a conventional approximation of $\bar{g}$ that is determined through the Poincare-Prey gravity reduction according to the general formula (ibid. p. 167)

$$
\bar{g}^{\text {helm }}=g+0.0424 H^{\text {helm }}
$$

where $g$ is the gravity value on the Earth's surface given in Gal and $H^{\text {helm }}$ is the Helmert orthometric height in $\mathrm{km}$; for more details see also Jekeli (2000). From the combination of Eqs. (2) and (3) the following equation is obtained

$$
h-N-H^{\text {helm }}=\frac{\bar{g}^{\text {helm }}-\bar{g}}{\bar{g}} H^{\text {helm }}+\frac{\delta W_{\mathrm{o}}}{g^{\left(W_{\mathrm{o}}\right)}}
$$

which corresponds to the rigorous constraint for geometric and Helmert orthometric heights in the presence of a geoid model. Other conventional types of orthometric heights (e.g. Niethammer, Mader) may also be used with Eq. (5) by modifying appropriately the scale factor of Eq. (3).

The above constraint emulates a transformation model between different vertical reference frames (VRFs) in the following sense

$$
H^{\mathrm{VRF} 2}-H^{\mathrm{VRF} 1}=\lambda H^{\mathrm{VRF} 1}+\mu
$$

where $\lambda$ is a differential scale factor relating the two frames and $\mu$ is the vertical offset of their zero-height reference surfaces. In our case, the first frame (VRF1) refers to the Helmert orthometric heights in a local vertical datum while the second frame (VRF2) is realized through the GPS derived orthometric heights with respect to a geoid model. Further remarks on the VRF transformation aspects related to the rigorous constraint in Eq. (5) will be given in Sect. 5.

\section{$4 \quad$ Theoretical Constraint for Geometric and Orthometric Heights in the Presence of a Quasi-geoid Model}

Let us consider the well-known theoretical relationship between geoid and quasi-geoid heights (Heiskanen and Moritz 1967, p. 326)

$$
N=\zeta+\frac{\bar{g}^{\prime}-\bar{\gamma}}{\bar{\gamma}} H^{\prime}
$$

where the (true) orthometric height $H^{\prime}$ refers to the geoidal equipotential surface $W=W_{\mathrm{o}}$ and it should be distinguished from the (true) orthometric height $H$ which was introduced in Eqs. (2)-(3) and refers to the equipotential surface
$W=W_{\mathrm{o}}^{\mathrm{LVD}}$ of a local vertical datum (see Fig. 1). The term $\bar{g}^{\prime}$ denotes the average gravity along the actual plumb-line between the Earth's surface and the geoidal equipotential surface, whereas $\bar{\gamma}$ is the average normal gravity along the normal plumb-line between the telluroid and the reference ellipsoid. For more details on the conversion between geoid and quasi-geoid heights, see Flury and Rummel (2009).

Starting from the rigorous integral expression that defines the average gravity $\bar{g}^{\prime}$, we have

$$
\begin{aligned}
\bar{g}^{\prime}= & \frac{1}{H^{\prime}} \int_{W=W_{o}}^{P} g d H \\
= & \left(\frac{1}{H^{\prime}} \int_{W=W_{o}}^{W=W_{o}^{\mathrm{LVD}}} g d H\right)+\left(\frac{1}{H^{\prime}} \int_{W=W_{o}^{\mathrm{LVD}}}^{P} g d H\right) \\
= & \frac{\delta W_{\mathrm{o}} / g^{\left(W_{\mathrm{o}}\right)}}{H^{\prime}}\left(\frac{1}{\delta W_{\mathrm{o}} / g^{\left(W_{\mathrm{o}}\right)}} \int_{W=W_{o}}^{W=W_{o}^{\mathrm{LVD}}} g d H\right) \\
& +\frac{H}{H^{\prime}}\left(\frac{1}{H} \int_{W=W_{o}^{\mathrm{LVD}}}^{P} g d H\right)=\frac{\delta W_{\mathrm{o}} / g^{\left(W_{\mathrm{o}}\right)}}{H^{\prime}} \bar{g}^{\left(\delta W_{\mathrm{o}}\right)}+\frac{H}{H^{\prime}} \bar{g}
\end{aligned}
$$

where $\bar{g}^{\left(\delta W_{\mathrm{o}}\right)}$ denotes the average gravity along the vertical segment between the equipotential surfaces $W=W_{\mathrm{o}}$ and $W=W_{\mathrm{o}}^{\mathrm{LVD}}$ while the rest of the terms were already introduced in the previous sections.

Substituting the result of Eq. (8) into Eq. (7) and considering that the orthometric heights $H$ and $H^{\prime}$ differ by the amount $\delta W_{\mathrm{o}} / g^{\left(W_{\mathrm{o}}\right)}$ (within a linear approximation), we have

$$
N=\zeta+\frac{\bar{g}-\bar{\gamma}}{\bar{\gamma}} H+\frac{\bar{g}^{\left(\delta W_{o}\right)}-\bar{\gamma}}{\bar{\gamma}} \frac{\delta W_{o}}{g^{\left(W_{o}\right)}}
$$

and by replacing $H$ according to Eq. (3), we obtain the relationship

$$
N=\zeta+\frac{\bar{g}^{\text {helm }}(\bar{g}-\bar{\gamma})}{\bar{g} \bar{\gamma}} H^{\text {helm }}+\frac{\bar{g}^{\left(\delta W_{o}\right)}-\bar{\gamma}}{\bar{\gamma}} \frac{\delta W_{\mathrm{o}}}{g^{\left(W_{\mathrm{o}}\right)}}
$$

Finally, if we combine the last equation with Eq. (5) we get the result

$$
h-\zeta-H^{\text {helm }}=\frac{\bar{g}^{\text {helm }}-\bar{\gamma}}{\bar{\gamma}} H^{\text {helm }}+\frac{\bar{g}^{\left(\delta W_{o}\right)}}{\bar{\gamma}} \frac{\delta W_{\mathrm{o}}}{g^{\left(W_{\mathrm{o}}\right)}}
$$

Since the equipotential surfaces $W=W_{\mathrm{o}}$ and $W=W_{\mathrm{o}}^{\mathrm{LVD}}$ do not deviate by more than few meters, it is reasonable to assume that $\bar{g}^{\left(\delta W_{0}\right)} \simeq g^{\left(W_{0}\right)}$ without causing any notable error in the last equation. More specifically, we have that

$$
\frac{\bar{g}^{\left(\delta W_{o}\right)}}{\bar{\gamma}} \frac{\delta W_{\mathrm{o}}}{g^{\left(W_{\mathrm{o}}\right)}}=\left(\frac{\bar{g}^{\left(\delta W_{o}\right)}-g^{\left(W_{\mathrm{o}}\right)}}{g^{\left(W_{\mathrm{o}}\right)}}\right) \frac{\delta W_{\mathrm{o}}}{\bar{\gamma}}+\frac{\delta W_{\mathrm{o}}}{\bar{\gamma}} \simeq \frac{\delta W_{\mathrm{o}}}{\bar{\gamma}}
$$


and thus Eq. (11) is practically equivalent to

$$
h-\zeta-H^{\text {helm }}=\frac{\bar{g}^{\text {helm }}-\bar{\gamma}}{\bar{\gamma}} H^{\text {helm }}+\frac{\delta W_{\mathrm{o}}}{\bar{\gamma}}
$$

The above relationship corresponds to a theoretical constraint for geometric and Helmert orthometric heights in the presence of a quasi-geoid model. It resembles a frame transformation model for physical heights in a similar sense as Eq. (6), where the first frame (VRF1) refers to the Helmert orthometric heights in a local vertical datum while the second frame (VRF2) is realized through the GPS derived normal heights with respect to a quasi-geoid model.

Interestingly enough, the terms $\bar{g}$ and $g^{\left(W_{\mathrm{o}}\right)}$ which appear in the geoid-related constraint of Eq. (5) (see previous section) have been now replaced by the mean normal gravity $\bar{\gamma}$. The latter is a computable quantity in terms of a truncated power series expansion of the station's normal height $H^{*}$ (Jekeli 2000, Eq. 29)

$$
\begin{aligned}
\bar{\gamma}= & \gamma\left(1-\left(1+f+\frac{\omega^{2} a^{2} b}{G M}\right.\right. \\
& \left.\left.-2 f \sin ^{2} \varphi\right) \frac{H^{*}}{a}+\left(\frac{H^{*}}{a}\right)^{2}+\ldots\right)
\end{aligned}
$$

where $\gamma$ is the normal gravity on the reference ellipsoid at the geodetic latitude $(\varphi)$ of the underlying station, $a, b$ and $f$ denote the length of the semi-major and semi-minor axes and the flattening of the reference ellipsoid, and finally $\omega$ and $G M$ correspond to the rotational velocity and the gravitational constant of the normal gravity field. In the context of Eq. (13), the required normal height $H^{*}$ for the implementation of the previous formula can be taken as the difference between the geometric height $(h)$ and the height anomaly $(\zeta)$ from the available quasi-geoid model.

\section{Discussion}

A modification of the well-known theoretical relationship between geometric and orthometric heights (see Eq. (1)) was presented in the preceding sections. The advantage of our revised formulation is that, instead of the non-determinable true orthometric heights, it employs the (most commonly used) Helmert orthometric heights in conjunction with a gravimetric geoid or quasi-geoid model. The corresponding constraints given in Eqs. (5) and (13) should be theoretically satisfied in the case of errorless data, and thus they provide a standard modeling framework for the joint analysis of heterogeneous heights in a terrestrial network.

Evidently, the height residuals $h-N-H^{\text {helm }}$ or $h-\zeta-H^{\text {helm }}$ will always contain a terrain-correlated part due to the difference of the "vertical metric" that is associated with the physical heights $H^{\text {helm }}$ and $h-N$ (or $h-\zeta$ ).
In reality, though, the terrain-correlated part of these residuals should not be attributed solely to the different vertical metrics as implied by the respective constraints (5) and (13), but it is also caused by hidden systematic errors which inflict an additional scale discrepancy among the heterogeneous height data.

Both of the derived constraints resemble a 1D similaritylike transformation between different vertical frame realizations; see Eq. (6). However, the height transformation parameters which are implied by the analytic forms of these constraints are not spatially invariant and thus Eq. (6) represents only a simplified approximation of the rigorous transformation between the underlying VRFs. More specifically,

- the spatial variation of the translation terms $\delta W_{\mathrm{o}} / g^{\left(W_{\mathrm{o}}\right)}$ and $\delta W_{o} / \bar{\gamma}$ is negligible even in large networks and their effect can be safely replaced by a mean vertical offset $\mu$, but

- the spatial variation of the scaling factors $\left(\bar{g}^{\text {helm }}-\bar{g}\right) / \bar{g}$ and $\left(\bar{g}^{\text {helm }}-\bar{\gamma}\right) / \bar{\gamma}$ on the Earth's surface may have a considerable contribution to the respective height residuals $h-N-H^{\text {helm }}$ and $h-\zeta-H^{\text {helm }}$, and it can create a significant nonlinear systematic behavior in their values.

Nonetheless, the simplified model in Eq. (6) can be effectively used for the least squares de-trending of heterogeneous height data and the assessment of their low-order systematic differences. Depending on the particular type of gravity field information that we have available (i.e. geoid or quasi-geoid heights), the following alternative forms of "observation equations" can be devised:

$$
\begin{aligned}
\left\{\begin{array}{c}
h_{i}-N_{i}-H_{i}^{\text {helm }} \\
h_{i}-\zeta_{i}-H_{i}^{\text {helm }}
\end{array}\right\}= & \left\{\begin{array}{c}
\lambda \\
\lambda^{\prime}
\end{array}\right\} H_{i}^{\text {helm }} \\
& +\delta W_{\mathrm{o}}\left\{\begin{array}{c}
1 / g^{\left(W_{\mathrm{o}}\right)} \\
1 / \bar{\gamma}_{i}
\end{array}\right\}+\left\{\begin{array}{c}
v_{i} \\
v_{i}^{\prime}
\end{array}\right\}
\end{aligned}
$$

which are deduced from Eqs. (5) and (13) by replacing the pointwise scaling factors with a "global" scale-change parameter. The terms $v_{i}$ and $v_{i}^{\prime}$ contain the total data noise and other systematic effects (including of course the spatial irregularities of the scaling factors $\left(\bar{g}^{\text {helm }}-\bar{g}\right) / \bar{g}$ and $\left(\bar{g}^{\text {helm }}-\bar{\gamma}\right) / \bar{\gamma}$ over the test network) which cannot be absorbed by the unknown parameters $\lambda$, or alternatively $\lambda^{\prime}$, and $\delta W_{0}$. Note that the latter will absorb not only the actual offset of the involved reference surfaces (i.e. $W \neq W_{\mathrm{o}}^{\mathrm{LVD}}$ ) but it will also be strongly affected by data systematic errors in spatial wavelengths that overly exceed the coverage of the test area. Anyhow, the results from the least squares adjustment of either version of Eq. (15) can support the conventional quality testing of global or regional gravity field models in GPS/leveling networks, as well as the assessment of the vertical frame consistency between the Helmert orthometric heights and the GPS/geoid orthometric heights (or the 
GPS/quasi-geoid normal heights) over a number of leveling benchmarks.

\section{Conclusions}

Our approach in this paper instigates a unified scheme with which the geometric and Helmert orthometric heights can be jointly analyzed and tested either with a geoid or quasi-geoid model. The second option may seem peculiar and in conflict with Molodensky's theoretical framework (Heiskanen and Moritz 1967, Chap. 8), yet it is fully justified on the basis of the fundamental constraint that was derived in Sect. 4. In fact, the direct combination of $h, \zeta$ and $H^{\text {helm }}$ according to Eq. (13) besides being theoretically valid, it is also practically advantageous since it avoids the conversion of the initially known height anomalies to geoid undulations (or, alternatively, the conversion of the orthometric heights to normal heights).

The aforementioned height conversion has been a prerequisite step for the consistent use of a quasi-geoid model with orthometric heights while its rigorous implementation is a challenging task especially in mountainous regions (e.g. Flury and Rummel 2009). Nevertheless, if Helmert orthometric heights are to be used then their consistent combination with a quasi-geoid model can be based on the much simpler equation (13) which essentially requires only the knowledge of:

- the surface gravity $g$ at each point (so that the term $\bar{g}^{\text {helm }}$ can be directly determined via Eq. (4)), and

- the geopotential difference $\delta W_{\mathrm{o}}$ between the local vertical datum of the Helmert orthometric heights and the $W_{\mathrm{o}}$ value which is implied in the zero-degree term of the quasi-geoid model.

Due to the limited extent of the current paper, it has not been possible to present some numerical examples related to the height modeling aspects that were discussed in the previous sections. A more thorough theoretical analysis, along with detailed examples using real data, will therefore be presented in a future journal paper.

\section{References}

Amos MJ, Featherstone WE (2009) Unification of New Zealand's local vertical datums: iterative gravimetric quasigeoid computations. J Geod 83:57-68

Burša M, Kenyon S, Kouba J, Šima Z, Vatrt V, Vojtišková M (2004) A global vertical reference frame based on four regional vertical datums. Stud Geophys Geod 48:493-502

Flury J, Rummel R (2009) On the geoid-quasigeoid separation in mountainous areas. J Geod 83:829-847

Fotopoulos G (2003) An analysis on the optimal combination of geoid, orthometric and ellipsoidal height data. PhD Thesis, UCGE Report No 20185, Department of Geomatics Engineering, University of Calgary, Calgary, Canada

Heiskanen W, Moritz H (1967) Physical geodesy. WH Freeman, San Francisco, CA

Jekeli C (2000) Heights, the geopotential and vertical datums. Department of Civil, Environmental Engineering and Geodetic Science, The Ohio State University, OSU Report No 459, Columbus, Ohio

Kotsakis C, Katsambalos K (2010) Quality analysis of global geopotential models at $1542 \mathrm{GPS} /$ leveling benchmarks over the Hellenic mainland. Surv Rev 42(318):327-344

Pan M, Sjöberg LE (1998) Unification of vertical datums by GPS and gravimetric geoid models with application to Fennoscandia. J Geod 72:64-70

Rapp RH (1994) Separation between reference surfaces of selected vertical datums. Bull Geod 69:26-31

Sánchez L (2007) Definition and realization of the SIRGAS vertical reference system within a globally unified height system. In: Tregoning P, Rizos Ch (eds) Dynamic planet. Springer, Berlin. IAG Symp 130:638-645 